\title{
Strategies for an Integrated Distribution Problem.
}

\author{
Rita Ribeiro* Helena R. Lourenço ${ }^{\dagger}$
}

\begin{abstract}
The problems arising in the logistics of commercial distribution are complex and involve several players and decision levels. One important decision is related with the design of the routes to distribute the products, in an efficient and inexpensive way.

This article explores three different distribution strategies: the first strategy corresponds to the classical vehicle routing problem; the second is a master route strategy with daily adaptations and the third is a strategy that takes into account the cross-functional planning through a multi-objective model with two objectives. All strategies are analyzed in a multi-period scenario. A metaheuristic based on the Iterated Local Search, is used to solve the models related with each strategy. A computational experiment is performed to evaluate the three strategies with respect to the two objectives. The cross functional planning strategy leads to solutions that put in practice the coordination between functional areas and better meet business objectives.

Keywords: Multi-objective VRP, Logistics, Iterated Local Search.

JEL: C61, C63, M19
\end{abstract}

\section{Introduction}

The growing number of problems that firms are facing nowadays with relation to the distribution of their products and services, has lead logistics to be of primary concern to many industries.

${ }^{*}$ Department of Economics and Business and GREL-IET, Universitat Pompeu Fabra, Ramon Trias Fargas 25-27, 08005 Barcelona (Spain). Tel: 34-93 5422692, Fax: 34-93 5421746. E-mail: rita.ribeiro@upf.edu. This research has been partially financed by research grant from Ministério da Ciência e a Tecnologia, FCT, Praxis XXI/BD/21814/99.

${ }^{\dagger}$ Department of Economics and Business and GREL-IET, Universitat Pompeu Fabra, Ramon Trias Fargas 25-27, 08005 Barcelona (Spain). Tel: 34-93 5422753, Fax: 34-93 5421746. E-mail: helena.ramalhinho@upf.edu. This research has been partially financed by research grant MCYT BEC-2000 and GREL-IET. 
An important aspect of the logistics management task is to coordinate the activities of the traditional distribution functions together with purchasing, materials planning, manufacturing, marketing and often R\&D. One important direction of the integration process is the cross functional planning, which consists of coordinating different areas inside the firm, allowing for cost reductions and improving service (see Christopher 1998).

The motivation of our work arises in this context of integration of logistics functions with other functions of the firm. In our case, we will focus our study in two key areas: the distribution management and marketing management.

On one hand, the importance of good distribution strategies in today's competitive markets can not be overstressed. In many industries, an important component of the distribution systems is the design of the routes of vehicles to serve their customer's demand. And, on the other hand, new trends in the supply-chain management are, as pointed out by some industry leaders, "..better customer service...greater customer sophistication" (OR/MS Today, August 2000). Customer service is becoming more important, customers demand more than a product, they demand a product arriving on time, an easy ordering system or a just-in-time distribution.

In this work, we will study the decision making problems in distribution management related with the delivery of products to customers, on a given period of time, using a fleet of vehicles. The decisions on how to assign customers to drivers and to design the routes made by each vehicle constitute the Vehicle Routing Problem (VRP). Usually, the VRP is responsibility of the Distribution department. However, since many drivers also perform commercial activities, the Marketing department has the objective of maintaining always the same delivery agent assigned to the same customer. How to balance these two, possible opposite, objectives is an interesting issue when the firm wants to implement integrated distribution processes.

To analyze the impact of integrating the two areas of the firm, Distribution and Marketing, we will explore three possible distribution strategies that reflect different policies in distribution in the organizations.

The first strategy, Strategy 1, has a distribution policy that minimizes distance. The objective is the classical objective of VRP, which consists of minimizing total routing cost, measured in distance units. However, this objective is often object of criticism by the users and planners, since it does not take into consideration other concerns of the company, for example, customer service. The second strategy, Strategy 2, tries to implement a marketing policy. In a growing competitive environment many firms adopt strategies of tight relationships with their customers where loyalty 
and friendship play a key role, through the delivering agents. In this strategy, the routes are predefined such that one delivering agent is associated with a specific set of customers.

The third strategy, Strategy 3, is the one that considers both objectives, marketing and distribution at the same time, in an integrated manner.

The motivation to work on this VRP arises by the distribution problems faced by the food and beverage industry. In these industries the tendency is to have lower inventories and higher delivery frequencies. And also, to have drivers responsible for commercial activities such as: promotions or introduction of new products.

The present article is organized in the following way: In the next section we will present the three distribution strategies. In Section 3, a review of the VRPs is summarized. Then, we will describe the mathematical formulation of each strategy and the solution approach, based on Iterated Local Search (ILS), to analyze those models. In section 5, we will present a computational experiment and analyze the results obtained. Finally, the conclusions of the work are presented.

\section{The Distribution Strategies}

The distribution strategies correspond to different situations and concerns inside the firm. By comparing them, we can analyze the effect that integrating two areas can have on the distribution policies. The objective of this analysis is to provide a set of possible alternative solutions to the decision maker. Whom, with the use of additional side information on each particular distribution problem, can make a good choice.

The strategies are settled in a planning horizon of a week, this is: five working days. The choice of this period is based on the need for a strategic perspective, we want to study the impact of a sequence of decisions on different objectives. As a consequence we need several periods to analyze the marketing effect and a week seems to be a reasonable choice since in many industries, the behavior of the orders for a customer have a week pattern (examples are the Beverage \& Food industry). In any case, this assumption can be relaxed and the problem could be extended to more periods.

\section{- Strategy 1: Distance Minimization}

In this strategy the distribution policy is constructed based on routing cost. Cost reduction is one of the biggest concerns in transportation and 
distribution management, but not the only one as we will see later. We want to find the route for each of the vehicles that will pass through the demand points in such a way as to satisfy all the demand with the smallest travelling cost. The classical VRP considers only one period at a time and chooses the optimal routes for that period. Strategy 1 corresponds to the classical vehicle routing problem (VRP) repeated for each day of the planning horizon.

\section{- Strategy 2: Master Routes}

The second strategy is based on marketing principles, and the distribution strategy is based on service measures. An important source of value to the firm can be obtained from a close connection between firm and customers. This can be achieved through the development and investment on the relationship with its customers.

In this strategy, each driver will serve always the same customer. This marketing policy is giving emphasis to the personal relationship between drivers and customers as a way to improve customer service. In many industries, drivers are more than drivers, they also perform sales tasks, promotions and introduction of the new products.

One of the advantages pointed out to this customer relationship management policy is that it makes more difficult for a customer to switch to another provider. Since, a relationship requires an investment of time from both the customer and the provider, Simchi-Levi et al. (2003). These marketing strategies allow the firm to obtain more information on customer needs. And, at the same time, it becomes more easy to introduce new products, construct promotions and even speeds up the delivery process due to experience effects from both parts.

\section{- Strategy 3: Multi-Objective}

The third strategy is the integrated distribution management model, which consists of taking into account in the decision process the concerns of the Distribution department and Marketing department i.e. the reduction of transportation costs and the emphasis on the personal relationship between driver and customer. We propose a multi-objective model with two objectives, each objective corresponds to a different function. The first is the transportation and the second a marketing function. This strategy tries to include in the same model the objectives of the two previous strategies. The best solution for the transportation problem might not always be the 
best solution for the marketing objective. In some cases these two objectives may conflict and that is the main justification for a trade-off analysis between these two objectives. We need to find a solution (or several solutions) that integrate a marketing policy and route design policy.

In the next section we will describe the mathematical model of each strategy.

\section{Literature Review on VRP}

The classical VRP model is behind the models for the three distribution strategies. Therefore, we make here a brief review of the classical VRP.

Vehicle routing decisions are extremely important within a company to maintain its competitiveness an allow it to best exploit the available resources and to distribute its products at the lowest possible cost.

Significant amount of research effort has been dedicated to VRP, see the survey articles on VRP by Laporte and Osman (1995), Laporte (1992), Desroisers et al. (1996), Bodin (1983), Cristofides (1979) and Fisher (1996) and the book of Crainic and Laporte(1998). An extensive list of VRP research papers can be found on http://www.imm.dtu.dk/ orgroup/VRP_ref/.

The most well known is a basic VRP which can be briefly defined in the following way: given a set of customers with known demand and location, define a set of routes, starting and finishing at one depot, that visits all customers with minimal cost.

The basic model of the vehicle routing problem considers a set of nodes, representing retailers or customers, at a known location, that must be served by one depot. Each node has a known demand. A set of vehicles $K$, with equal capacity is available to serve the customers. The routes must start and finish at the depot. The objective is to define the set of routes to serve all customers with minimal cost.

For each pair of nodes, a fixed known cost is associated. We assume this cost matrix is symmetric and can represent a real cost, distance or time. The main constraints of the problem are that all the demand must be satisfied and the vehicles capacity can not be exceeded.

The basic VRP is a generalization of the Traveling Salesman Problem, where more than one vehicle is available, for TSP references see for example Lawer et al.(1985).

There are several formulations of the classical VRP in the literature, for some of these formulations see Fisher and Jaikumar (1978) (1981), Kulkarni and Bhave (1985), Gouveia (1995) and Toth and Vigo (2002). 
The classical model of the VRP can be formulated as an integer linear programming and this is the formulation we will use along further chapters.

Consider the following data:

$I=1, \ldots, n \quad$ set of nodes, that correspond to the different locations of the customers, node 1 corresponds to the depot.

$K=1, \ldots, m \quad$ set of vehicles;

$Q \quad$ capacity of each vehicle;

$q_{i} \quad$ demand of customer $i, i=1, \ldots, n$;

$c_{i j} \quad$ cost of going from $i$ to $j, i=1, \ldots, n ; j=1, \ldots, n$.

This formulation considers two types of variables:

$x_{i j k}= \begin{cases}1, & \text { if vehicle } k \text { visits customer } j \text { immediatly after customer } i \\ 0, & \text { otherwise }\end{cases}$

$y_{i k}= \begin{cases}1, & \text { if customer } i \text { is visited by vehicle } k \\ 0, & \text { otherwise }\end{cases}$

The formulation of the problem is: 
Objective Function :

$\operatorname{Min} \sum_{i=1}^{n} \sum_{j=1}^{n} c_{i j} \sum_{k=1}^{m} x_{i j k}$

Subject to:

$$
\begin{aligned}
& \sum_{k=1}^{m} y_{i k}=1, \quad \forall i=2, \ldots, n \\
& \sum_{k=1}^{m} y_{1 k}=m, \\
& \sum_{i=2}^{n} q_{i} y_{i k} \leq Q, \quad \forall k=1, \ldots, m \\
& \sum_{j=1}^{n} x_{i j k}=\sum_{j=1}^{n} x_{j i k}=y_{i k}, \\
& \forall i=2, \ldots, n ; k=1, \ldots, m \\
& \sum_{j, i \in S} x_{i j k} \leq|S|-1, \\
& \forall S \text { non-empty subset of }\{2, \ldots, n\} ; k=1, \ldots, m \\
& x_{i j k} \in\{0,1\} ; y_{i k} \in\{0,1\}, \\
& \forall i=1, \ldots, n ; k=1, \ldots, m
\end{aligned}
$$

Constraint (2) ensures that each customer is visited by one vehicle only. Constraint (3) guarantees that all vehicles visit the depot. Constraint (4) represents the vehicle capacity constraint. For each vehicle $k$, we guarantee that the sum of the demand of the nodes that the vehicle covers is less or equal to its maximum capacity. Here we assume that none of the customers has a daily demand that exceeds $Q$. The constraint (5) ensures that if a vehicle visits a customer it also has to leave that customer.

Constraint (6) is the sub-tour elimination constraint. This constraint implies that the arcs selected contain no sub-cycles. It states that for every vehicle, the following holds: for every non-empty subset $S$ of $\{2, . ., n\}$, the number of arcs that are in the route of this vehicle, with both nodes belonging to $S$, has to be less or equal to the number of elements of $S$ minus 1.

The last constraint, (7) defines the variables $x$ and $y$ as binary. The objective function is minimizing the total cost of the routes. 
The TSP is a sub-problem of the VRP, the TSP belongs to the class of $N P$-hard (non-deterministic polynomial time) problems, and so does the basic VRP and extensions. This means that the computational complexity of the problem grows exponentially with its size, i.e., it grows exponentially with the number of customers.

\section{The Models for the Distribution Strategies}

In this section we will present the mathematical models associated with the three strategies. First of all, we describe the assumptions of the model.

We assume that the firm is responsible for the distribution of its own products. Therefore, there are no questions of outsourcing to be handled. These firms face the pressures of a competitive market making them concerned both on consumer satisfaction and internal efficiency.

The classical VRP considers only one period and chooses the optimal routes for that period. Here we will introduce more periods by considering a week length of analysis. Each day we have a different set of customers to serve and quantities to deliver. The reduction of the inventory levels, and the increase in the frequency of orders is a tendency in many businesses to lower stock handling costs.

Other assumptions of the model are:

- All the demand is satisfied in the same day that it is required and not on any other day of the week.

- Only unload is done at each customer.

- The number of vehicles is fixed and there are no fixed costs associated with the use of the vehicles. They all have the same capacity. Moreover, the number of vehicles available is enough to satisfy all the demand.

- Another assumption is that the distance matrix between customers and between customers and warehouse is known and fixed. This matrix does not depend on the day or the quantity loaded.

- Each vehicle is assigned to a driver. And we consider that they work every day in the period in question.

- One vehicle can only be used once a day and the time it takes to deliver the full capacity is less than a working day. 
Next we will present the model in detail. The following data is considered in the mathematical formulation:

$i, I$ index and set of nodes, $I=1, \ldots, n$ where 1 is the depot and 2 to $n$ are the customers locations;

$k, K$ index and set of vehicles, $K=1, \ldots, m$;

$t, T$ index and set of days which represent the period, $T=1, \ldots, p$;

$T_{i}$ set of days where customer $i$ has a demand that is greater than zero, $i=2, \ldots, n$;

$q_{i}^{t}$ demand of customer $i$ on day $t, i=1, \ldots, n$ and $t=1, \ldots, p$;

$c_{i j}$ the cost of going from $i$ to $j$, this is a fixed matrix $, i=1, \ldots, n$ and $j=1, \ldots, n$;

$Q$ capacity of a vehicle.

The variables of the model are:

$x_{i j k}^{t}= \begin{cases}1, & \text { if vehicle } k \text { visits customer } j \\ 0, & \text { otherwise }\end{cases}$

$y_{i k}^{t}= \begin{cases}1, & \text { if customer } i \text { is visited by vehicle } k \text { on day } t \\ 0, & \text { otherwise }\end{cases}$

\subsection{Strategy 1: Distance Minimization}

The objective function minimizes routing costs, for all customers during the week period. This strategy corresponds to repeating a classical VRP for each day of the week.

The formulation of this objective will be the same as the one used for the classical model but with a new parameter, $t$, representing the day of the week.

$$
\text { Objective function: } \quad \operatorname{Min} \sum_{t=1}^{p} \sum_{i=1}^{n} \sum_{j=1}^{n} \sum_{k=1}^{m} c_{i j} x_{i j k}^{t}
$$




\subsection{Strategy 2: Master Routes}

First we construct a set of routes, "master routes", using the same VRP formulation as in Strategy 1.

This startegy and the model associated is very close to a common practice in several companies. It consists in: first defining "master routes" and, afterwards performing daily adjustments depending on the demand of the customer and on the capacity of the vehicle. To obtain the "master routes" we consider a VRP model, where all customers are in the input data and the demand of each customer depends on the average daily demand. To adjust the daily routes we consider other constraints such as capacity and number of vehicles.

So, the requirement that a customer will always be served by the same driver may have to be sacrificed but we will try to enforce this at least to the best customers. Therefore, the idea is: the better the customer is the more interest we have in maintaining the same driver.

The mathematical formulation for this strategy is identical to the one of the classical VRP for one period presented in section 2, but, in this case all customers are considered for the "master routes".

\subsection{Strategy 3: Multi-Objective}

In this strategy we propose a multi-objective model with two objectives: minimizing of routing costs and minimization of service levels, that reflect the integrated strategies between Distribution and Marketing departments.

As far has we know there are no studies on routing problems with multiple periods and this marketing oriented objective function. Although there are some multi-objective VRP that consider other types of objectives. Examples of these are the papers by Hong and Park (1999) that consider the minimization of the customer waiting time as the second objective function, in a VRP with time windows constraints. Lee and Ueng (1999) developed an integer linear model that searches for the shortest travel path and balances driver's load simultaneously. The work is measured in terms of traveling and loading time. The objective function is the weighted sum of the above objectives. This second objective minimizes the difference between the working time of each vehicle and the working time of the vehicle with the shortest working time.

In most cases of multiple objectives it is unlikely that the problem is optimized by the same alternative parameter choices. Hence, some trade-off between the criteria is needed to ensure a satisfactory design. 
In the multi-objective optimization an important relation is the dominance relation.

Let $\left(z_{1}\right)$ and $\left(z_{2}\right)$ be two solutions of a multi-objective problem with $R$ objectives.

We say that:

Solution $\left(z_{1}\right)$ dominates $\left(z_{2}\right) \quad$ if $\quad z_{1 r} \leq z_{2 r} \quad \forall$ objectives $r$ in $\{1, \ldots, R\}$ and $z_{1 r}<z_{2 r}$ for at least one $r$ and $\left(z_{1}\right) \neq\left(z_{2}\right)$.

A feasible solution is efficient if it is non-dominated.

Based on this concept we will optimize the two objective functions to find non-dominated solutions.

Ideally, we would like to find the solution that would be optimal for both objectives at the same time. In multi-objective programming this solution point rarely exists. So, we would like to find solutions that are closer to this ideal point.

Mathematically, all dominated solutions are equally acceptable, it is the decision maker, who is responsible for choosing the final solution. The decision maker is someone who has a deep knowledge of the problems, relationships and implications of each solution. The choice among these non-dominated solutions is determined by the decision maker's preferences among the multiple objective.

The two objective functions considered within the integrated strategy are:

\section{Objective A: Minimizing Cost}

The formulation of this objective will be the same as in (8), the one used for the model of strategy 1.

$$
\text { Objective function A: } \quad \operatorname{Min} \sum_{t=1}^{p} \sum_{i=1}^{n} \sum_{j=1}^{n} \sum_{k=1}^{m} c_{i j} x_{i j k}^{t}
$$

\section{Objective B: Marketing Objective}

In terms of mathematical formulation, the second objective works as follows: For each customer we have a set of pairs of days with positive demand, $T_{i}$, for each pair of days $g, h$ in $T_{i}$ (with $g \neq h$ ) we want to 
minimize the difference in the assignment to a vehicle $k$. The objective is to minimize $\left|y_{i k}^{g}-y_{i k}^{h}\right|$.

The importance is given by the total demand in the period, therefore a weight is introduced by the total amount ordered by each customer. The objective function becomes:

$$
\operatorname{Min}\left\{\sum_{i=1}^{n} \sum_{\substack { k=1 \\
\begin{subarray}{c}{g, h \in T_{i} \\
g<h{ k = 1 \\
\begin{subarray} { c } { g , h \in T _ { i } \\
g < h } }\end{subarray}}^{m}\left[\left(\sum_{t=1}^{p} q_{i}\right) \times\left|y_{i k}^{g}-y_{i k}^{h}\right|\right]\right\}
$$

The importance of a customer is measured in terms of sales. In some cases other measures could be used to classify the goodness of a customer, for example, frequency of orders, credit history, etc. This function is non linear.

Considering a multi-period model is an essential point in our study since, objective B is not static, it measures decisions allong more than one period, it only makes sense in a continued time base.

In the integrated strategy the objective is to find a set of non-dominated solutions and give the decision maker the possibility to choose not only between strategies but also between solutions.

The constraints of the model for strategy 1 and 3 are: 


$$
\begin{aligned}
& \sum_{k=1}^{m} y_{i k}^{t}=1, \forall i=2, \ldots, n ; t \in T_{i} \\
& \sum_{k=1}^{m} y_{i k}^{t}=m, \forall t \in T ; i=1 \\
& \sum_{i=2}^{n} q_{i}^{t} y_{i k}^{t} \leq Q, \forall k=1, \ldots, m ; t=1, \ldots, p \\
& \sum_{j=1}^{n} x_{i j k}^{t}=\sum_{j=1}^{n} x_{j i k}^{t}=y_{i k}^{t}, \\
& \forall i=2, \ldots, n ; k=1, \ldots, m ; t=1, \ldots, p \\
& \sum_{k=1}^{m} y_{i k}^{t}=0, \forall i=2, \ldots, n \text { and } t \notin T_{i} \\
& \sum_{j, i \in S} x_{i j k}^{t} \leq|S|-1, \\
& \forall S \text { nonempty subset of }\{2, \ldots, n\} ; \\
& k=1, \ldots, m ; t=1, \ldots, p \\
& x_{i j k}^{t} \in\{0,1\} ; y_{i k}^{t} \in\{0,1\} \\
& \forall i=1, \ldots, n ; k=1, \ldots, m ; t=1, \ldots, p
\end{aligned}
$$

Constraints (9) to (15) are similar to the ones in the basic model, but for each day of the period in question.

Constraint (9) ensures that in the days where the customers have a positive demand, that customer is visited by only one vehicle.

The constraint (10) forces that each day all vehicles go to the depot. Constraint (11) ensures that, the daily loading of a vehicle does not exceed its capacity.

Constraint (12) guarantees that if the vehicle enters a node, on day $t$, it also has to leave that node, on the same day.

Constraint (13) prohibits a vehicle to visit a customer on a day where he has zero demand.

Finally constraint (14) avoids sub-tours, but now not only for each vehicle but also for each day. The sub-tour elimination constraint represents an exponential number of constraints. 
The last constraint (15) define all variables as binary.

For Strategy 2 the constrains of the model are the same as for VRP formulation in section 2, applied in this occasion to the "master routes", only for one period.

\section{Solution Approach}

The above models are complex combinatorial optimization problems, classified as $N P$-hard ${ }^{1}$. The effort required to solve $N P$-hard problems increases exponentially with problem size. The approach for solving these type of problems optimally suffers from computational burden with problem size. As a result they require an heuristic methodology in order to solve them. Since our objective is to compare the three distribution strategies we will use the same solution technique for each strategy.

\section{$5.1 \quad$ Heuristics method}

A heuristic algorithm is a solution method that does not guarantee an optimal solution, but in general had a good level of performance in term of solution quality and convergence. Heuristics may be constructive (producing a single solution) or local search (starting from one given random solutions and moving iteratively to other nearby solutions) or combination. Heuristics for VRP have been extensively studied. Cordeau et. el. (2002) summarize the most important classical and modern heuristics for the VRP. Osman and Laporte (1996) have a bibliography review on theory and application of metaheuristics.

Local search is the most powerful general approach for finding high quality solutions to hard combinatorial optimization problem in reasonable time.

It is based on the iterative exploration of neighborhoods of solutions trying to improve the current solution by local changes. The type of local search that may be applied to a solution is defined by a neighborhood structure.

The most basic local search is the iterative improvement. It typically starts with an initial solution, generated randomly or by some constructive heuristic and tries to repeatedly improve a current solution by moves to better neighboring solutions. A major drawback of iterative improvement

\footnotetext{
${ }^{1} \mathrm{NP}$-hard problems are the class of network and combinatorial problems for which no polynomial-bounded algorithm has yet been found.
} 
local search is that it may stop at very poor quality local minimum. To avoid the disadvantage of iterative improvement and, in particular, multiple descendent, we need to allow the local search to escape from local optima. To escape from stagnation at local optima is the main goal of methaheuristics. Metaheuristics are typically high level strategies, which guide an underlying, more problem specific heuristics, to increase their performance.

Our proposal is to use a metaheuristic algorithm that as proven to give quiet good results on other problems and is easy to implement and modify, adapting to different strategies, Iterated Local Search (ILS).

\subsubsection{Iterated Local Search for the VRP}

ILS is a simple and generally applicable meta-heuristic which iteratively applies local search to modifications of the current search point, for more detailed information on ILS see Lourenço et. al. (2001), Lourenço et. al. (2002) and Stützle (1998). At the start of the algorithm a local search is applied to some initial solution. Then, a main loop is repeated until a stopping criterion is satisfied. This main loop consists of a modification step ("perturbation"), which returns an intermediate solution corresponding to a modification of a previously found locally optimal solution.

Next, local search is applied to yielding a locally optimal solution . An "acceptance criterion" then decides from which solution the search is continued by applying the next "perturbation". Both, the perturbation step and the acceptance test may be influenced by the search history. ILS is expected to perform better than if we just restart local search from a new randomly generated solutions.

The architecture of the ILS is as follows: 


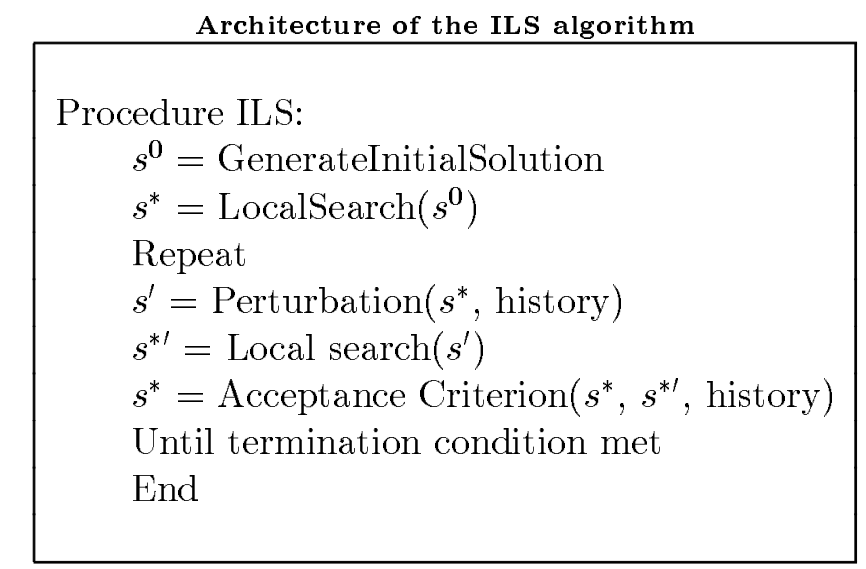

The proposed ILS heuristics is based on the ILS metaheuristic developed by Stützle (1998) and Kunz (2000) to solve the classical VRP. The ILS used for the VRP is the following:

ILS for the VRP

Step 1. Savings Heuristic - Initial Solution

Step 2. ILS for TSP on each tour:

Step 2.1. Local Search for TSP

Step 2.2. Perturbation for TSP

Step 2.3. Acceptance criterion

Step 3. ILS for the VRP

Step 3.1. LS for the Assignment Problem

Step 3.2. Perturbation for VRP

Step 3.3 Acceptance Criterion

Step 4. ILS for the TSP on the new routes

We will now present the implementation of each step the above algorithm in more detail.

\section{- Savings Heuristic}

This is a greedy heuristic to construct an initial solution. It has been proved that starting from a random solution gives worst results (Stützle 1998). This savings heuristic gives us the tours to start the search, it was proposed by Clarke and Wright (1962).

\section{- ILS for the TSP}


On each of the tours obtained in the savings heuristic we apply an ILS. At this step of the algorithm we ignore any relation between routes.

\section{LS for TSP}

The LS used was a 2-opt, this is: on a tour 2 connections are exchanged as soon as all customers are tested with the others, we have a new constellation, since there are now new crusts that have not been checked, the process is repeated. Only when a complete run without improvements finishes, one has reached a 2-opt resolution.
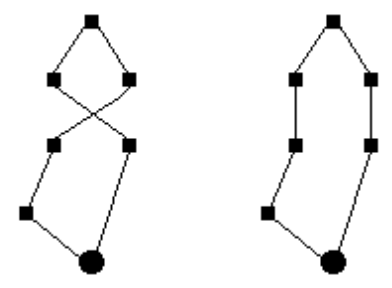

Figure 1: Example of a 2-opt move for the TSP

Searching in a complete 2-opt would not be efficient. So, to reduce the search space, some techniques are introduced that faster the process and still arrive to good quality solutions: A list of candidates and "don't look bits". One "don"t look bit" is associated with each node. Initially, all "don't look bits" $=0$, if for a node no improving move can be found, then "don't look bit" is turned on (set to 1) and is not considered as a starting node in the next iteration. If an edge incident to a node is changed by a move, the node's "don't look bit" is turned off again - reduces to $O(n)$.

Perturbation (Kick-move) for TSP

On the local minimum that has been reached, we apply the kick-move and arrive to a new start solution. The goal here is to escape from local optima by applying perturbations to the current local minimum.

For the LS on the TSP we use "double bridge", this perturbations cuts four edges, and introduces 4 new ones.

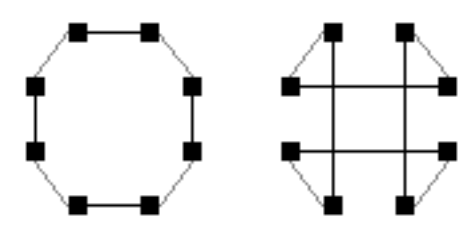

Figure 2: Example of a Double Bridge move. 
Acceptance criterion

The acceptance criterion used at this step is better; this means that the new tour is accepted if it has a lower cost.

\section{- ILS for the VRP}

The ILS for the VRP is implemented considering the initial solution the routes obtained from the ILS of the TSP.

LS for the assignment problem

The local search for the VRP is a 2-opt and again a list of candidates and "don't look bits" techniques are applied to restrict the search.

We have two possibilities for a 2-opt: A customer of a tour is postponed into another or a customer trades with another customer from another tour. First, if capacity restrictions allow and it reduces costs, a city is inserted in the tour. Only if it cannot be inserted, then we check if an exchange with another tour improves the solution.
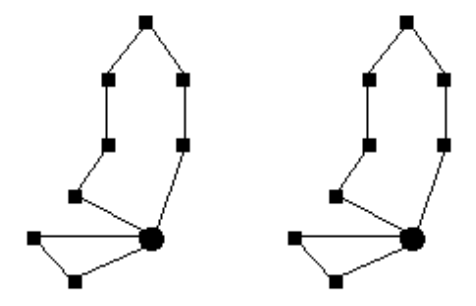

Figure 3: Example of a 2-opt move for the VRP

The same techniques as in LS for the TSP are used: "don't look bit" and List of candidates.

Kick-moves

"Numb-crosser": This perturbation consists in exchanges a group of customers from 2 tours. In this case, $1 / 3$ of the customers of the tour are exchanged.

Acceptance criterion: Best, the same as the acceptance criterion for the TSP.

\section{- ILS for the TSP on the new routes}

Repeats the ILS procedure for the TSP. 


\subsubsection{The ILS for each strategy}

The ILS for the VRP is now adapted to solve the 3 models for the different strategies. Next we will describe in detail th ILS for each of them.

Strategy 1: Since in this strategy we have a classical VRP model, for each day, we apply the ILS to find the best daily routes, according to the capacity of the vehicle and daily demand. The algorithm is repeated for several runs and for each day chooses the best solution.

Let $L$ be the total number of loops:

\section{Structure of the algorithm for Strategy 1}

Step 1: Set loop $=0$

Step 2: Set day $=1$

Step 3. Savings Heuristic - Initial Solution

Step 4. ILS for TSP on each tour

Step 5. ILS for the VRP

Step 6. ILS for the TSP on the new routes

Step 7. Set $d a y=d a y+1$; Repeat Step 3 to 6 until day $=5$;

Step 8. Set loop $=$ loop +1

Step 9. Repeat Step 2 to 8 until loop $=L$

Strategy 2: In this strategy, we have considered a classical VRP model to obtain the "master routes", where all customers are taken into account using its average demand. Therefore, to obtain the master routes we apply an ILS.

Afterwards, the routes, for each day of the week, are obtained in the following way: Exact the same routes are maintained for each day; Eliminate from the routes the customers that have no demand on that day; If in any of the routes the capacity constraint is being violated, we pick up from the tour the customer that is considered less important and we delete it from this tour and insert it on another tour. This tour is chosen in such a way as to minimize routing costs. 
Structure of the algorithm for Strategy 2

Step 1: For all customers do

Step 1.1. Savings Heuristic - Initial Solution

Step 4. ILS for TSP on each tour

Step 5. ILS for the VRP

Step 6. ILS for the TSP on the new routes

Step 2: Set $d a y=1$

Step 3. For each tour eliminte customers with zero demand

Step 4: For each tour, if capacity constraints are violated

remove customer with lowest total demand

Step 5: ILS for the TSP on the new routes

Step 6: Set $d a y=d a y+1$

Step 7: Repeat Step 3 to 6 until day $=5$;

Strategy 3: In this strategy we face a multi-objective combinatorial optimization problem(MOCOP). Ehgoot and Gandibleux (2000) provide an annotated bibliography on MOCOP.

Two main approaches can be found in the metaheuristics for the MOCOP: methods of local search (LS) in object space and population based methods. In the LS methods, we start from an initial solutions, and the procedure approximates a part of the non-dominated frontier corresponding to the search direction $\lambda$ given. A local aggregation mechanism of the objectives, based on the weighted sum, produces the effect to focus the search on a part of the non-dominated frontier. The principle is repeated for several search directions. In the population based methods, all population contributes to the evolution process toward the non dominated frontier. Here we will use the first approach, i.e. methods based on local search.

In this case, after having decided the routes for the first day, the program takes into consideration objectives $\mathrm{B}$, through a wheighted function of both objectives. To do this, we calculate the effect of a move on the weighted function of the objectives. Then, in the acceptance criterion, a new solution is accepted if the weighted function has improved. The algorithm is repeated for several different sets of weights. All the non-dominated solutions are keep during the run of the algorithm.

A objective function $Z$ is used as weighted function, which is a weighted sum of the single objectives $A$ and $B$. 
Let $f_{r}$ be the single objective function of objective $r$,

$$
Z=\sum_{r=1}^{2} w_{r} f_{r} \text { and } \sum_{r=1}^{2} w_{r}=1
$$

The solution is very sensitive to the weights that have been defined. The problem lies also on having objectives with different variables and scales. In our case, for example we are adding costs and quantities.

Notation:

$w_{a}$ weight for Objective A, with $0 \leq w_{a} \leq 1$;

$w_{b}$ weight for Objective B, with $0 \leq w_{b} \leq 1$;

and

$$
\begin{aligned}
& w_{a}+w_{b}=1 \\
& z=w_{a}(\text { Objective } A)+w_{b}(\text { Objective } B)
\end{aligned}
$$

Structure of the algorithm for Strategy 3

Step 1: Set $w_{a}=1$ and $w_{b}=0$

Step 2: Set $d a y=1$

Step 3. Savings Heuristic - Initial Solution

Step 4. ILS for TSP on each tour

Step 5. ILS for the VRP

Step 5.1. LS for the Assignment Problem

Step 5.1.1 For each move calculate the effect on objective $A$ and $B$

Step 5.1.1 Accept only if the new $\mathrm{z}$ is smaller

Step 5.2. Perturbation for VRP

Step 5.3. Repeat Step 3.1

Step 5.3 Acceptance Criterion

Step 6. ILS for the TSP on the new routes

Step 7. Set $d a y=d a y+1$; Repeat Step 3 to 6 until $d a y=5$;

Step 8. Set $w_{a}=w_{a}-0.1$ and $w_{b}=w_{b}+0.1$

Step 9. Repeat Step 2 to 8 until $w_{a}=1$ and $w b=0$ 


\section{Computational experiment}

The main objective of this experience is to evaluate the three strategies and analyze the effect of each objective in the solutions. With this purpose we have applied the above algorithms on several sets of randomly generated examples. The results are expressed in terms of the values of the objectives and total number of vehicles needed. For each strategy two values were calculated: the Routing Cost and the Marketing (or service) Value (these are the two objectives of strategy 3). The first is measured in distance and the second can be interpreted as the unit cost for the distributor for not serving a customer with the same salesman, working in a similar way as penalty cost.

Next we will explain the data used and analyze some important results of this experiment.

\subsection{The data}

For the computational experiment, we have generated several sets of examples concerning the total number of customers $(50,100,200,400)$. Also, we have examples with two types of demand (low variation and high variation) and two types of vehicles capacity, high and low.

To obtain the demand, we have used a normal distribution with mean 50 and standard deviation 20 for the case demand has a high variation and a standard deviation of 5 for the examples with low variation. For each day, on average, $25 \%$ of the customers have zero demand. This implies that in a problem of 100 customers, with 5 days week, we will have around 375 deliveries to make.

The customers locations are uniformly generated in a $100 \times 100$ square with the depot located in the center with the coordinates $(50,50)$.

Truck capacity is 300 for problems of sizes 50, 100, 200 and 700 for size 400. Also run cases with size 200 and capacity 500. In total we have studied 30 examples for each strategy, therefore we will consider 90 problems per run.

\subsection{Analysis of the results}

In this section we will present the results obtained for each example in terms of the objective functions values, number of vehicles used, non-dominated solutions and run times.

We can start illustrating the aim of the different strategies by looking 
at a small example with 2 days and a few customers: in Figure 4 we have the routes for two days, in strategy 1,2 and 3. Strategy 1 has fewer and more efficient routes in terms of distance, Strategy 2 has more routes, but the routes are the same for each day. And, Strategy 3, has a solutions that is not completely efficient in terms of distance, but allowing for a better service level.

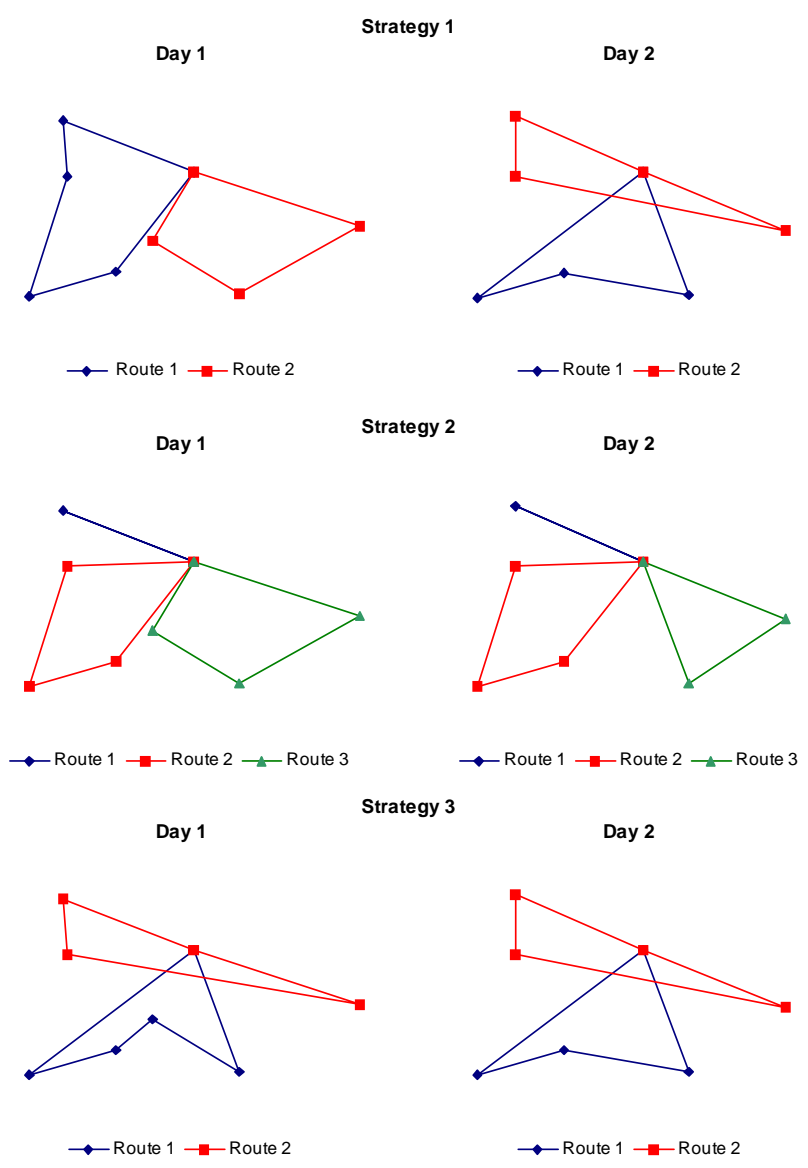

Figure 4: Routes for Strategy 1, 2 and 3.

Table 1 and 2 show the results for each example and for each strategy. Strategy 1 tries to find lowest cost, strategy 2 the best service level and strategy 3 the set of non-dominated solutions with respect to the integrated strategy. We can observe that, as expected, strategy 1 will always give us the solution with the lowest objective $A$ and the highest objective $B$ when 
compared with strategy 2 . In the strategy 2 we have much lower marketing values but the cost of routes increases significantly.

Concerning Strategy 3, we can say that, in almost every example, we can find more than one non-dominated solution. In case 4, for example we have 4 non-dominated solutions and it would be the responsibility of the decision maker to decide on one of the alternatives. 


\begin{tabular}{|c|c|c|c|c|c|c|c|}
\hline \multirow[b]{2}{*}{$N$} & \multirow[b]{2}{*}{ Example } & \multicolumn{2}{|c|}{ Strategy 1} & \multicolumn{2}{|c|}{ Strategy 2} & \multicolumn{2}{|c|}{ Startegy 3} \\
\hline & & a & $b$ & $a$ & b & $a$ & $b$ \\
\hline \multirow[t]{17}{*}{50} & 1 & 14344,15 & 36840 & 17018,9326 & 3306,00 & 14510,3408 & 32327 \\
\hline & & & & & & 14344,1455 & 36840 \\
\hline & 2 & 13589,47 & 38538 & 16304,18 & 13589,4697 & 13755,11 & 35826 \\
\hline & & & & & & 13697,92 & 38148 \\
\hline & & & & & & 13589,47 & 38538 \\
\hline & & & & & & 13680,72 & 38278 \\
\hline & 3 & 13225,97 & 40284 & 15744,37 & 4997 & 13289,63 & 36178 \\
\hline & & & & & & 13228,96 & 40284 \\
\hline & & & & & & 13278,06 & 40140 \\
\hline & 4 & 11710,85 & 32341 & 15597,20 & 5950 & 11850,30 & 25753 \\
\hline & & & & & & 11811,55 & 31776 \\
\hline & & & & & & 11710,85 & 32341 \\
\hline & & & & & & 11788,70 & 32289 \\
\hline & 5 & 13452,19 & 38084 & 16832,33 & 3012 & 13541,62 & 30733 \\
\hline & & & & & & 13462,15 & 38426 \\
\hline & & & & & & 13473,14 & 38198 \\
\hline & & & & & & 13485,40 & 37925 \\
\hline \multirow[t]{18}{*}{100} & 6 & 22970,35 & 75096 & 28356,94 & 8368 & 23240,29 & 64967 \\
\hline & & & & & & 23033,34 & 75012 \\
\hline & & & & & & 22971,54 & 75096 \\
\hline & & & & & & 23162,88 & 74404 \\
\hline & & & & & & 23153,67 & 74601 \\
\hline & 7 & 21999,94 & 73497 & 27755,47 & 7209 & 22045,08 & 61244 \\
\hline & & & & & & 22041,77 & 73416 \\
\hline & & & & & & 21999,94 & 73497 \\
\hline & 8 & 21839,25 & 74834 & 26873,79 & 10264 & 21907,99 & 63623 \\
\hline & & & & & & 21839,25 & 74834 \\
\hline & 9 & 17062,82 & 70744 & 24874,82 & 5045 & 17236,27 & 59365 \\
\hline & & & & & & 17036,71 & 70838 \\
\hline & & & & & & 17220,50 & 69385 \\
\hline & & & & & & 17167,73 & 70579 \\
\hline & 10 & 22383,01 & 76252 & 27892,61 & 5987 & 22632,56 & 63871 \\
\hline & & & & & & 22467,32 & 76110 \\
\hline & & & & & & 22493,46 & 75632 \\
\hline & & & & & & 22462,16 & 76252 \\
\hline \multirow[t]{9}{*}{200} & 11 & 40438,9102 & 152536 & 52738,0898 & 19418 & 40682,9297 & 132320 \\
\hline & & & & & & 40438,9102 & 152536 \\
\hline & & & & & & 40484,59 & 152292 \\
\hline & 12 & 40420,20 & 152564 & 52089,76 & 9967 & 40757,39 & 134118 \\
\hline & & & & & & 40420,20 & 152564 \\
\hline & 13 & 38484,12 & 151187 & 49881,28 & 19803 & 38498,67 & 131916 \\
\hline & & & & & & 38423,13 & 150677 \\
\hline & 14 & 38481,27 & 148056 & 47837,33 & 9999 & 38681,92 & 129944 \\
\hline & & & & & & 38710,30 & 147051 \\
\hline
\end{tabular}

Table 1: Routing Cost (a) and Marketing Level (b) for Strategy 1, 2 and 3. 


\begin{tabular}{|c|c|c|c|c|c|c|c|}
\hline \multirow[b]{2}{*}{$N$} & \multirow[b]{2}{*}{ Example } & \multicolumn{2}{|c|}{ Strategy 1} & \multicolumn{2}{|c|}{ Strategy 2} & \multicolumn{2}{|c|}{ Strategy 3} \\
\hline & & $a$ & $b$ & a & $b$ & a & $b$ \\
\hline 200 & 16 & 28589,23 & 152040 & 35849,68 & 8375 & 28883,37 & 132744 \\
\hline \multirow[t]{20}{*}{$(\mathrm{cap}=500)$} & & & & & & 28782,42 & 151361 \\
\hline & & & & & & 28780,13 & 151569 \\
\hline & & & & & & 28686,55 & 151883 \\
\hline & & & & & & 28551,02 & 152164 \\
\hline & & & & & & 28630,92 & 152040 \\
\hline & 17 & 28675,59 & 150844 & 34171,21 & 2096 & 28814,46 & 130891 \\
\hline & & & & & & 28711,49 & 150542 \\
\hline & & & & & & 28704,59 & 150844 \\
\hline & 18 & 27350,06 & 149576 & 33919,55 & 9715 & 27511,86 & 130867 \\
\hline & & & & & & 27474,54 & 149315 \\
\hline & & & & & & 27255,56 & 150108 \\
\hline & & & & & & 27503,72 & 149261 \\
\hline & & & & & & 27397,52 & 149435 \\
\hline & & & & & & 27371,87 & 149576 \\
\hline & 19 & 27674,9883 & 147608 & 33632,543 & 9637 & 27676,8105 & 128579 \\
\hline & & & & & & 27613,74 & 147754 \\
\hline & 20 & 27836,23 & 152388 & 34002,28 & 9320 & 28035,45 & 130763 \\
\hline & & & & & & 27896,09 & 151456 \\
\hline & & & & & & 27824,33 & 151509 \\
\hline & & & & & & 27990,09 & 151355 \\
\hline \multirow[t]{13}{*}{400} & 21 & 40013,37 & 304388 & 49637,54 & 9607 & 40120,91 & 260814 \\
\hline & & & & & & 40101,29 & 304184 \\
\hline & & & & & & 40023,38 & 304388 \\
\hline & 22 & 39758,16 & 308956 & 47691,43 & 9401 & 39961,71 & 270449 \\
\hline & & & & & & 39845,06 & 308604 \\
\hline & & & & & & 39842,24 & 308956 \\
\hline & 23 & 39576,79 & 300057 & 47635,00 & 6954 & 39658,17 & 255775 \\
\hline & & & & & & 39584,53 & 299568 \\
\hline & 24 & 39949,22 & 302980 & 47978,58 & 9525 & 40022,79 & 266906 \\
\hline & & & & & & 39956,93 & 303057 \\
\hline & & & & & & 39970,21 & 302980 \\
\hline & 25 & 39552,16 & 299976 & 46682,13 & 9538 & 39593,31 & 264664 \\
\hline & & & & & & 39583,24 & 299976 \\
\hline 50 & 26 & 12606,09 & 37756 & 11465,43 & 2032 & 12870,39 & 33258 \\
\hline \multirow[t]{9}{*}{ (low stdev) } & & & & & & 12716,2236 & 37124 \\
\hline & & & & & & 12606,09 & 37756 \\
\hline & & & & & & 12867,78 & 36802 \\
\hline & 27 & 13752,04 & 37759 & 11913,72 & 0 & 13861,01 & 34403 \\
\hline & & & & & & 13715,64 & 37757 \\
\hline & & & & & & 13855,72 & 37428 \\
\hline & 28 & 12723,78 & 39888 & 11096,46 & 0 & 12820,78 & 33940 \\
\hline & & & & & & 12781,623 & 39888 \\
\hline & 29 & 12468,2988 & 32983 & 11225,9092 & 0 & 12571,04 & 25854 \\
\hline
\end{tabular}

Table 2: Routing Cost (a) and Marketing Level (b) for Strategy 1, 2 and 3. 
In Figure 2 we can see the set of all solutions obtained for 22 iterations for an example of 50 customers. The blue squares correspond to the dominated solutions and the red squares correspond to non-dominated solutions.

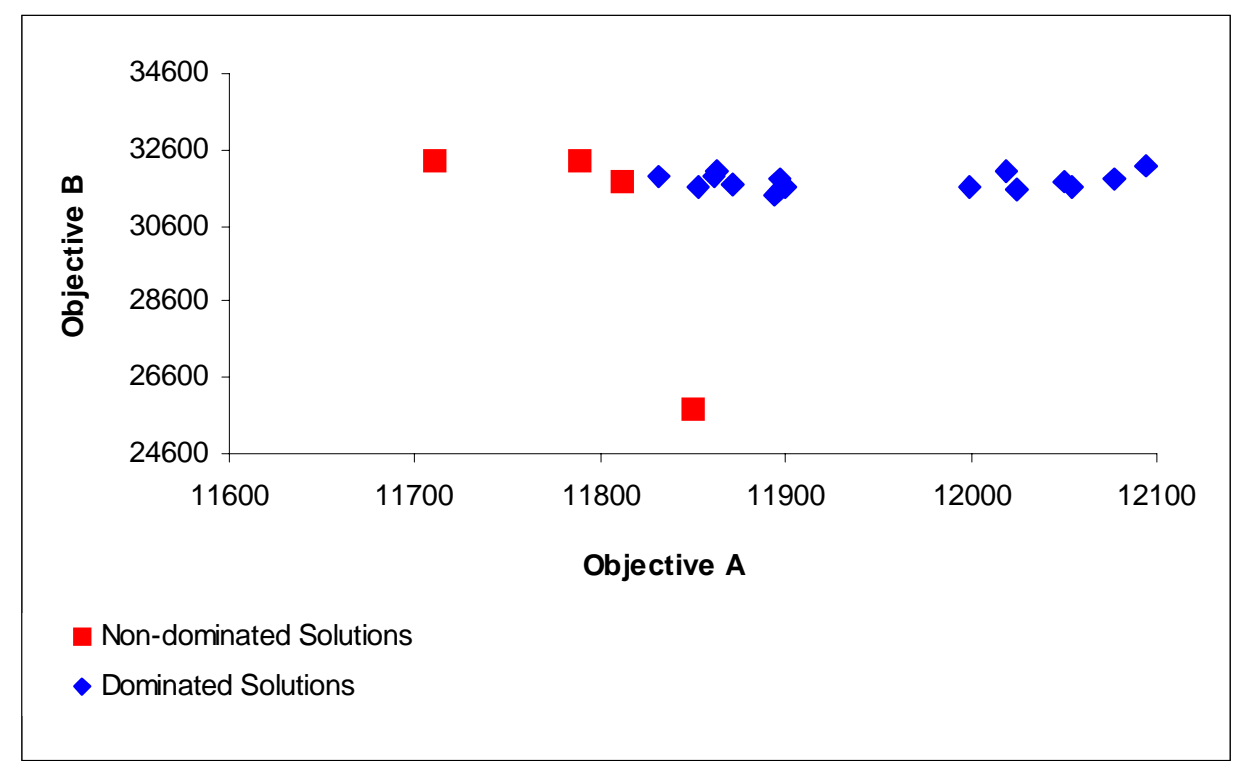

Figure 5: Example of a set of dominated and non-dominated solutions for Strategy 3.

The number of vehicles needed for each solution strategy also varies and this is reflected in the total distance cost. In Table 3, we can observe these differences. The master routes approach has always much higher number of vehicles a week, this happens due to the procedure of the routes design, they are constructed considering all customers, and then for each day eliminating the ones with no demand. When constructing the "master routes" we have used the daily average demand of each customer. Nonetheless, if we had chosen higher values for the demand, associated with each customer, the higher the number of vehicle used in the "master routes" and the lower the marketing objective.

Comparing the number of vehicles from Strategy 1 and Strategy 3 we observe that, on average, Strategy 3 has the same or a higher number of vehicles, this is due to the existence of the second objective, that introduces a preference for service rather than distance. To have a better service we 


\begin{tabular}{lccc}
\hline $\boldsymbol{N}$ & Strategy & Strategy 2 & Strategy 3 \\
\hline \hline 50 & 36 & 44 & 36 \\
100 & 68 & 84 & 68 \\
200 & 135 & 167 & 135 \\
200 (cap $=500)$ & 80 & 99 & 81 \\
400 & 114 & 141 & 114 \\
50 (low stdev) & 36 & 26 & 36 \\
\hline
\end{tabular}

Figure 1: Table 3: Average number of vehicles needed per week, for Strategy 1,2 and 3.

need to sacrifice the routing efficiency and this can pass through the use of an additional vehicle.

In terms of running time, the importance of the results can be overstressed. The first and third strategies are the ones that take more time to compute. But, since we are referring to strategic planning, it does not seem inefficient for a firm, with a network of 400 customer, to spend one hour on a strategic planning task for a week. Take into consideration that in many cases the week planning pattern is repeated in the next weeks. Table 4 summarizes running times. For Strategy 1 and 3 we have done the same number of iterations. The magnitude of the difference in run time of strategy 2 is due to the fact that we only run for once the VRP, for the master case. On the other two strategies we have to run VRP for each day of the panning period several times. 


\begin{tabular}{lccc}
\hline $\boldsymbol{N}$ & Strategy $\mathbf{1}$ & Strategy 2 & Strategy 3 \\
\hline \hline 50 & 146,19 & 2,32 & 142,40 \\
100 & 383,95 & 6,24 & 384,38 \\
200 & 1116,73 & 17,09 & 1076,41 \\
200 (cap $=500)$ & 1497,82 & 21,13 & 1409,40 \\
400 & 3199,07 & 29,07 & 3088,93 \\
50 (low stdev) & 152,59 & 3,05 & 149,16 \\
\hline
\end{tabular}

Table 4: Average run time in seconds, per problem size, for Strategy 1, 2 and 3.

Finally, in Table 5 we show the results of other versions of algorithm for strategy 3 . In version 2 we have introduced more iterations for each weight. And, in version 3 we have done more iterations for the ILS for each day, and kept the same number of iterations per weight. From the results we can conclude that by allowing more running time, the algorithm of version 2 , on average, gives more non-dominated solutions in 3 of the 5 problems. In version 3 , on average the number of non-dominated solutions is smaller than in the other versions but we are able to improve the solutions, when comparing with version 1 and 2 . 


\begin{tabular}{|c|c|c|c|c|c|c|}
\hline \multirow[b]{2}{*}{ Examples } & \multicolumn{2}{|c|}{ Startegy 3, version 1} & \multicolumn{2}{|c|}{ Startegy 3, version 2} & \multicolumn{2}{|c|}{ Startegy 3 , version 3} \\
\hline & $a$ & $b$ & $a$ & $b$ & $a$ & $b$ \\
\hline \multirow[t]{3}{*}{1} & 14510,34 & 32327 & 14510,34 & 32327 & 14372,34 & 32327 \\
\hline & 14344,15 & 36840 & 14376,34 & 36570 & 14251,39 & 36840 \\
\hline & & & 14318,05 & 36840 & & \\
\hline Run Time & 151,60 & & 369,65 & & 406,61 & \\
\hline \multirow[t]{5}{*}{2} & 13755,11 & 35826 & 13755,11 & 35826 & 13657,49 & 35826 \\
\hline & 13697,92 & 38148 & 13697,92 & 38148 & 13631,61 & 38148 \\
\hline & 13589,47 & 38538 & 13680,72 & 38278 & 13944,69 & 39060 \\
\hline & 13680,72 & 38278 & 13644,05 & 38376 & & \\
\hline & & & 13589,47 & 38538 & & \\
\hline Run Time & 141,22 & & 358,72 & & 386,46 & \\
\hline \multirow[t]{3}{*}{3} & 13289,63 & 36178 & 13289,63 & 36178 & 13235,85 & 35668 \\
\hline & 13228,96 & 40284 & 13224,70 & 40284 & 13229,69 & 40148 \\
\hline & 13278,06 & 40140 & & & 13183,31 & 40284 \\
\hline Run Time & 150,23 & & 378,33 & & 398,76 & \\
\hline \multirow[t]{4}{*}{4} & 11850,30 & 25753 & 11850,30 & 25753 & 11812,68 & 25393 \\
\hline & 11811,55 & 31776 & 11805,80 & 31416 & 12126,23 & 31416 \\
\hline & 11710,85 & 32341 & 11751,11 & 32062 & & \\
\hline & 11788,70 & 32289 & 11710,85 & 32341 & & \\
\hline Run Time & 123,64 & & 311,10 & & 329,89 & \\
\hline \multirow[t]{4}{*}{5} & 13541,62 & 30733 & 13541,62 & 30733 & 13515,69 & 30336 \\
\hline & 13462,15 & 38426 & 13485,40 & 37925 & 13441,13 & 36998 \\
\hline & 13473,14 & 38198 & 13452,19 & 38084 & 13364,65 & 38084 \\
\hline & 13485,40 & 37925 & & & & \\
\hline Run Time & 145,33 & & 368,83 & & 397,50 & \\
\hline
\end{tabular}

Table 5: Routing Cost (a) and Marketing Level (b) for the non dominated solutions of Strategy 3, for 3 different versions of the algorithm.

\section{Summary and Conclusions}

In this paper we have explored different distribution strategies to analyze an integrated distribution problem. The strategies cover a week planning horizon and reflect different ways of looking at the distribution problem. The first strategy is the classical VRP approach, which reflects only transportation cost: For each day of the planning horizon the routes are designed minimizing routing costs. The second strategy is a more customer oriented strategy based on customer relationship management principle, where mas- 
ter routes are constructed to ensure a marketing policy where each customer is always served by the same driver. The third strategy is a multi-objective combinatorial optimization problem with two objectives: minimizing cost and improving customer service. This third strategy results from the integration of the two other strategies and brings together two important areas in many industries: Distribution and Marketing. The idea was to compare this new approach with the other two strategies.

For each of the above strategy we have presented a mathematical model and a heuristic procedure, based on the ILS, to solve the problems. Then, the three algorithms were applied to a set of randomly generated instances. The main conclusion is that the multi-objective model gives several nondominated solutions, that can be seen as a good balance between optimizing the transportation cost or customer service. The decision maker has to choose the solution which meets better his business needs, since cost minimization is not the only concern in distribution management.

There are several possible extensions of this work, one is in the area of the metaheuristics and here it would be interesting to develop multi-objective population based metaheuristic to solve the multi-objective model and to perform a comparison with the current approach. The second extension would be to include other objectives that would reflect different business needs, as for example, the one of balancing the routes. This is particularly interesting if we assume that driver's remuneration is related with truck loading. In this case, we would be studying the integration decisions with the Human Resources department.

\section{References}

[1] Bodin, L. (1983). "Routing and Scheduling of Vehicles and Crews, The state of the art." Computers \& Operations Research 10: 63-211.

[2] Christofides, N., A. Mingozzi, et al. (1979). The Vehicle Routing problem. Combinatorial Optimization. N. Christofides, A. Mingozzi, P. Toth and Saudi. New York, John Wiley \& Sons: 315-338.

[3] Christopher, M. (1998). Logistics and Supply Chain Management: Strategies for reducing cost and improving service, Financial Times, Prentice Hall. 
[4] Clarke, G. and J. W. Wright (1964). "Scheduling of vehicles from a central depot to a number of delivery points." Operations Research 12: $568-581$.

[5] Cordeau, J. F., G. Laporte, et al. (2002). "A guide to vehicle routing heuristics." Journal of the Operational Research Society 53: 512-522.

[6] Crainic, T. G. and G. Laporte (1998). Fleet Management and Logistics. Centre for Research on Transportation, Kluwer Academic Publishers.

[7] Desrosiers, J. ,Dumas, Y., Solomon, M.M., and Soumis, F. (1996), "The routing and Scheduling" in Networks and Distribution, Handbooks in OR and MS, North-Holland, Amsterdam.

[8] Ehrgott, M. and X. Gandibleux (2000). An Annotated Bibliography of Multiobjective Combinatorial Optimization, Universitat Kaiserslautern.

[9] Fisher, M.L., Jaikumar (1978), "A decomposition algorithm for largescale vehicle routing", report 78-11-05, Department of decision Sciences, The Wharton School, University of Pennsylvania, Philadelphia $[12 ; 2,3]$.

[10] Fisher, M.L., Jaikumar (1981), "A generalized assignment heuristic for vehicle routing", Networks 11, 109-124[12:2,4].

[11] Fisher, M. L. (1995). Vehicle Routing. Network Routing. M.O.Ball, T. L. Magnanti, C. L. Monma and G. L. Nemhauser. Amsterdam, NorthHolland. 8: 1-33.

[12] Gouveia, L. (1995), "A result on projection for the Vehicle Routing Problem", European Journal of Operational Research 85, 610-624.

[13] Hong, S.-C. and Y.-B. Park (1999). "A heuristic for bi-objective vehicle routing with time windows constraints." International Journal of Production Economics 62: 249-258.

[14] Kulkarni, R. V. and P.R.Bhave (1985). "Integer programming formulations of vehicle routing problems." European Journal of Operational Research 20: 58-67.

[15] Kunz, C. (2000). Iterierte Lokale Suche ur das Vehicle-Routing Problem, TU Damstadt. 
[16] Laporte, G. (1992). "The Vehicle Routing Problem: An overview of exact and approximate algorithms." European Journal of Operational Research 59: 345-358.

[17] Laporte, G. and Osman, I.H. (1995), "Routing problems: A bibliography", Annals of Operations Research.

[18] Laporte, G. and I. H. Osman (1996). "Metaheuristics: A bibliography." Annals of Operations Research 63: 513-623.

[19] Lawer, E.L., Lenstra, J.K., Rinnooy Kan, A.H.G. and Shmoys, D.B. (1985), "The Traveling Salesman Problem", Wiley \& Sons Ltd.

[20] Lee, T. and Ueng, J. (1999), "A study of vehicle routing problems with load-balancing", International Journal of Physical Distribution 83 Logistics Management, Vol.29, No.10, 646-658.

[21] Lourenco, H., Martin, O. and Stützle T. (2001), “A Beginner's Introduction to Iterated Local Search.", In Proceedings of MIC 2001, pages 1-6, Porto, Portugal, July 2001.

[22] Lourenco, H., Martin, O. and Stützle T. (2002), "Iterated Local Search.", In F. Glover and G. Kochenberger, editors, Handbook of Metaheuristics, pages 321-353, Kluwer Academic Publishers, Norwell, MA.

[23] Partyka, J. G. and R. W. Hall (2000). "On the Road to Service". OR/MS Today(August 2000): 26-35.

[24] Simchi-Levi, D., P. Kaminsky, et al. (2003). Designing and Managing the Supply Chain: Concepts, Strategies and Case Studies, MacGrawHill, Irwin.

[25] Stützle, T. G. (1998). Local Search Algorithms for Combinatorial Problems - Analysis, Improvements, and New Applications. Department of Computer Science, Darmstadt University of Technology.

[26] Toth, P. and D. Vigo (2002). "Models, relaxations and exact approaches for the capacitated vehicle routing problem." Discrete Applied Mathematics 123: 487-512. 